\title{
HIGH REDSHIFT GALAXIES
}




\title{
THE EVOLUTION OF FIELD GALAXIES
}

\author{
S.J. LILLY \\ University of Toronto, Toronto, Canada \\ O. LE FEVRE AND F. HAMMER \\ Observatoire de Paris, Meudon, France \\ D. CRAMPTON \\ Dominion Astrophysical Observatory, Victoria, Canada \\ D.J. SCHADE AND J.D. HUDON \\ University of Toronto, Toronto, Canada \\ AND \\ L. TRESSE \\ University of Cambridge, Cambridge, UK
}

\section{Introduction}

During the late 1980 's, successively deeper redshift surveys carried out with multi-object spectrographs on 4-m class telescopes produced growing evidence for evolution in the galaxy population. While some evolution had been expected from analysis of the galaxy number counts, the surprising indication from the first deep redshift surveys was that this appeared to involve moderate luminosity galaxies lying at moderate redshifts (Broadhurst et al. 1988, Colless et al. 1990, Cowie et al. 1991). However, while the results were suggestive, these early surveys suffered a number of significant problems that hampered their interpretation:

(a) the samples were small, especially at the faintest levels, so the statistical weight was limited and analysis was based on crude parameterizations of the data such as the median redshift of samples;

(b) the typical redshifts were small $(z<<0.5)$, so that evolutionary effects could only be seen against "local" populations whose selection was often quite different - indeed the local luminosity function of galaxies is still poorly defined (Loveday et al. 1992, Marzke et al. 1994); 
(c) the samples were selected in the observed $B$-band, so that comparison with local samples was based on the poorly constrained ultraviolet properties and relative numbers of galaxies of different types.

As reviewed by Koo and Kron (1992), the evidence for there being any evolution was thus not completely watertight, and as recently as 1993, Koo et al. (1993) were able to produce unevolving models for the galaxy population which were consistent with the available $B$-band faint galaxy data.

In order to improve on this situation, two independent French and Canadian programs (Tresse et al. 1993, Lilly et al. 1993) merged into the CanadaFrance Redshift Survey (CFRS). We adopted the ambitious observational goal of securing spectroscopic identifications for $1000 \mathrm{I}$-band selected objects with $17.5<I_{A B}<22.5$. The $I$-band selection was chosen to correspond to selection at rest-wavelengths between $B$ and $V$ for $0.5<z<0.9$, and the depth was chosen to yield a median redshift $\langle z\rangle \sim 0.6$, corresponding to a look-back time of $50 \%$ of the age of the Universe (for $\Omega=1$ ).

\subsection{THE CANADA-FRANCE REDSHIFT SURVEY}

The data and methods of the Canada-France Redshift Survey (CFRS) have been described in detail elsewhere (Lilly et al. 1995a, Le Fevre et al. 1995a, Lilly et al. 1995b, Hammer et al. 1995a, Crampton et al. 1995). The final "statistically-complete" sample consists of 943 objects: 591 galaxies with secure redshifts $0.0<z<1.3$ and a median $<z>=0.55,6$ quasars, 200 stars and 146 objects (believed to be nearly all galaxies) that were unidentified or had insecure spectroscopic identifications.

The CFRS was designed to allow three separate approaches to be taken to studying the evolution of galaxies and large scale structure over the last 2/3 of the Hubble time. Specifically, we designed the program:

(a) To define the basic statistical properties of the galaxy population, such as the luminosity function $\phi(L$, colour,$z)$ and the spatial correlation function, $\xi(r, z)$, over the redshift range $0<z<1$, using samples selected in as similar a way as possible to local samples of galaxies.

(b) To produce large well-defined sub-samples of the galaxy population which could then be studied in more detail (spectroscopically, morphologically, kinematically) to reveal the most important physical processes occurring in galaxies at earlier epochs, and thus to link the evolution of individual galaxies to the changes seen in the population as a whole.

(c) To relate faint field galaxies (the "normal" population) to objects selected in deep surveys at other wavelengths (e.g. the radio, infrared and millimeter wavebands. As an example, Hammer et al. (1995b) discuss the identifications of a large well-defined sample of $\mu$-Jy radio sources. 


\section{Statistical descriptions of the galaxy population at high red- shift}

\subsection{THE LUMINOSITY FUNCTION}

Fig 1 shows the luminosity function $\phi(L$, colour, $z)$ over the interval $0<$ $z<1.3$ (computed in the rest-frame $B$-band for $q_{0}=0.5$ and $H_{0}=50$ $\mathrm{kms}^{-1} \mathrm{Mpc}^{-1}$ ). The most remarkable feature of this diagram is the clear difference between the evolution of the red and blue populations of galaxies (split at the colours of the Coleman et al. (1980) Sbc spectral energy distribution). It should be stressed here that the luminosity function, of course, reflects changes in the population and not (neccessarily) changes in individual objects.

The luminosity function of the population of red galaxies shows virtually no change (for $\Omega=1$ ) all the way back to $z \sim 1$. This suggests that the population of massive, reasonably quiescent galaxies, was to a very large degree "in place" at $z \sim 1$. Interestingly, we see no evidence for significant luminosity evolution (of order 0.8 magnitudes) that might be expected from the passive evolution of the stellar population. However, the expected passive luminosity evolution depends sensitively on the slope of the stellar initial mass function as well as the age (Tinsley and Gunn, 1976). Changes of a few tenths of a magnitude in $\mathrm{L}^{*}$ of the red population back to $z \sim 0.8$ are certainly not ruled out (Lilly et al., 1995c) and adoption of a lower value of $q_{0}$ would also imply greater evolution. An improved estimate of the expected passive luminosity evolution would greatly help in the interpretation of the red luminosity function.

The luminosity function of bluer galaxies shows a substantial enhancement at $z>0.5$ in the numbers of galaxies with roughly present-day $\mathrm{L}^{*}$ $\left(M_{A B}(B) \sim-21.0\right)$. This evolving blue population, now clearly revealed in the luminosity function is responsible for steepening the galaxy number counts down to $B \sim 24$. The observed changes in the blue luminosity function between $z \sim 0.3$ and $z \sim 0.7$ could be due to either (a) a uniform brightening of all blue galaxies by about 1.5 magnitudes, (b) an increase in the number density of galaxies of constant luminosity by a factor of three, (c) a combination of both effects, or (d) an even more complicated scenario involving galaxies crossing the red-blue divide. Further information on the environments, morphologies, kinematics, and stellar populations of the galaxies (see below) should allow us to discriminate between these scenarios and will lead to an understanding of the physical evolution of individual galaxies as well as the statistical evolution of the population as a whole. 


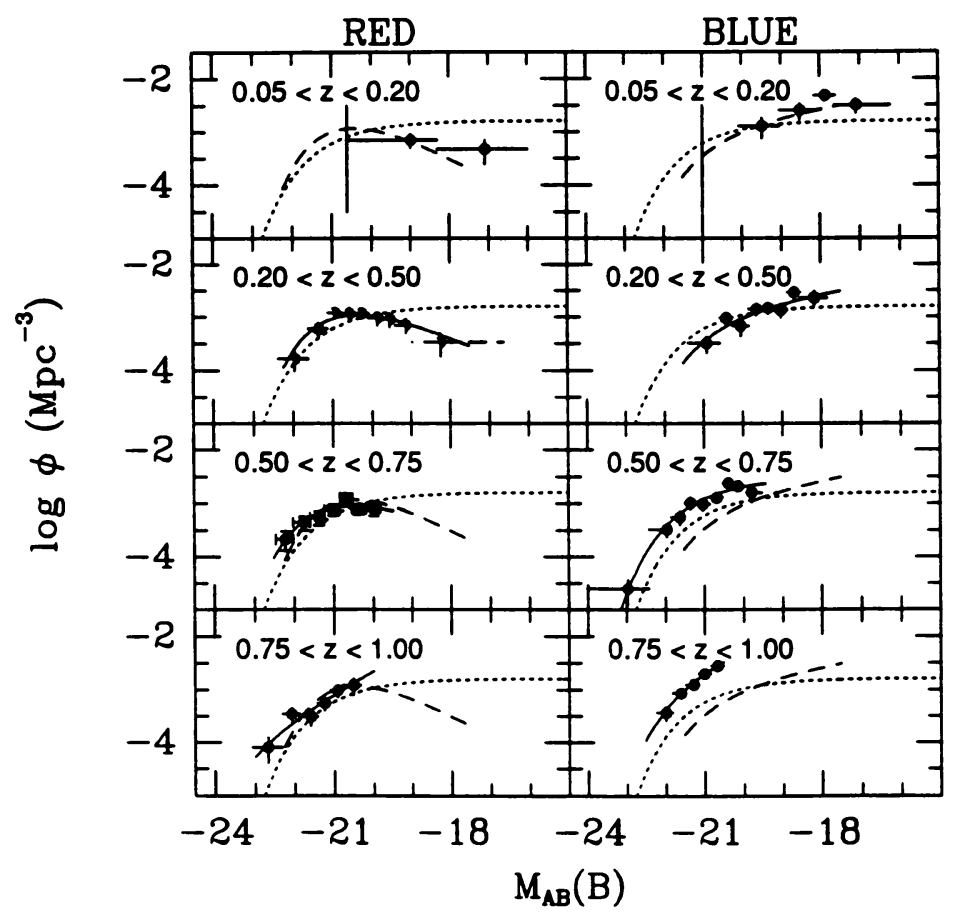

Figure 1. The CFRS luminosity function $\phi(L$, colour, $z)$ split by intrinsic colour and redshift (computed for $H_{0}=50 \mathrm{kms}^{-1} \mathrm{Mpc}^{-1}$ and $q_{0}=0.5$ ). This shows that the luminosity function of red galaxies changes very little over the interval $0<z<1$, while the luminosity function of blue galaxies changes significantly at $z>0.5$. See Lilly et al. (1995c) for details.

\subsection{THE CLUSTERING CORRELATION FUNCTION}

The evolving amplitude of the correlation function $\xi(r, z)$ reflects both the growth of large scale structure and the nature of the faint galaxy population, since the population at high redshift may be differently clustered (intrinsically) to that at low redshift. The strength of galaxy clustering at high redshift has been studied with the CFRS in two ways: (a) from the redshift catalogue itself (Le Fevre et al., 1996) using the $w\left(r_{p}\right)$ formulism (Davis and Peebles, 1983) and (b) by using the observed overall $N(z)$ to invert the projected two-dimensional correlation function $w(\theta)$ obtained for much larger samples of galaxies (Hudon and Lilly, 1996). These two approaches give consistent results (see Fig 2).

The physical correlation length $r_{0}(z)$ that is measured (Fig 2) may be parameterized as 


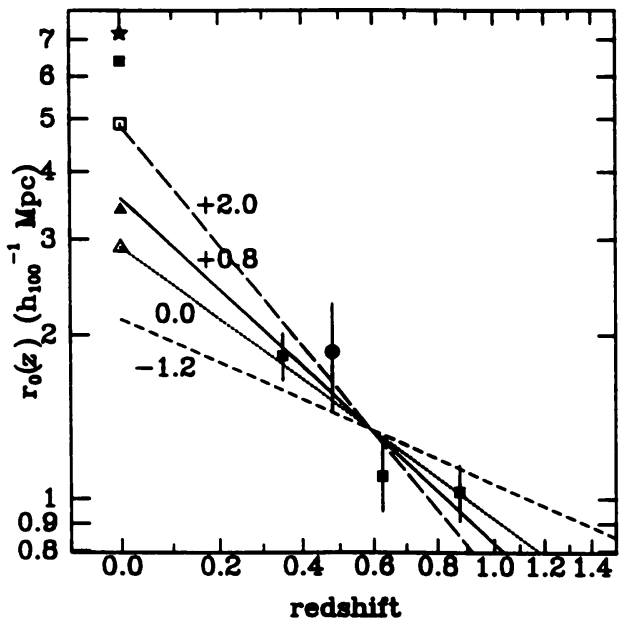

Figure 2. The scale length of the correlation function, $r_{0}(z)$ at high redshift as derived directly from the CFRS sample (solid squares) and from the projected $w(\theta)$ and the CFRS $\mathrm{N}(\mathrm{z})$ (solid disk) (computed for $H_{0}=100 \mathrm{kms}^{-1} \mathrm{Mpc}^{-1}$ and $q_{0}=0.5$ ). The diagonal lines indicate various evolutionary models parameterized and labelled by the $\epsilon$ parameter. If the faint galaxy population is to evolve into the local population of massive galaxies seen in the CfA or IRAS samples, then evolution with $\epsilon \geq+0.8$ is required. See Le Fevre et al (1996) for details.

$$
r_{0}(z)=r_{0}(0)(1+z)^{-(3+\epsilon) / \gamma}
$$

with $\gamma=1.8$ as the slope of the power-law correlation function. If clustering does not grow (i.e. is fixed in comoving space) $\epsilon=\gamma-3 \sim-1.2$. A scenario in which clustering is represented as bound systems in an expanding Universe has $\epsilon=0$, while the mass-distribution in CDM-like hierarchical models has $\epsilon \sim+0.8$ on these scales.

If, as seems reasonable (from the luminosity function results, above, and the imaging results, below), the galaxy population at high redshift evolves into something like the local CfA sample, with $r_{0} \sim 5.5 h_{100}^{-1} \mathrm{Mpc}$ (Davis and Peebles, 1983), or the $60-\mu \mathrm{m}$-selected IRAS sample of star-forming galaxies, with $r_{0} \sim 3.7 h_{100}^{-1} \mathrm{Mpc}$ (Fisher et al., 1994) then we require, for $q_{0} \sim 0.5$, strong growth of clustering, with $+0.8<\epsilon<+2.0$. A scenario involving no growth of structure would produce a local population that would be much more weakly clustered than any local population. For $q_{0} \sim 0$, less evolution is required and evolution to an IRAS-like sample is consistent with the $\epsilon=0$ "stable clustering" growth case.

An important result (Le Fevre et al., 1996) is the demonstration that the red and blue populations at $z>0.5$ have very similar auto-correlation (and also cross-correlation) function amplitudes. Thus the spatial segregation between blue and red galaxies seen locally (and seen at marginal significance 
in our own data at $z<0.5$ ) has largely disappeared at $z>0.5$. This may be seen as an analogous effect to the Butcher-Oemler effect.

\section{Towards a physical interpretation of galaxy evolution}

\subsection{GALAXY MORPHOLOGIES FROM HST IMAGING}

The high number density of sources at $I_{A B} \sim 22.5$ means that each WFPC2 field in our redshift survey areas contains many galaxies with spectroscopically determined redshifts. To date, we have images in F450W and F814W of four WFPC2 fields from the CFRS and a resulting sample of 32 galaxies at $z>0.5$ ((Schade et al., 1995)). This should increase sixfold within a year or so.

The 32 galaxies at $z>0.5$ show the full range of Hubble types from ellipticals through spirals to irregulars. Spiral arms and bars are clearly visible in many galaxies and 4 of the 32 clearly look as if they are merging/interacting galaxies. As expected, the galaxies are considerably less regular on the shorter wavelength images.

Our analysis has centered on two-dimensional fitting of the galaxies using bulge+disk models and examination of the subsequent residual images (Schade et al., 1995). About $70 \%$ of the galaxies form a regular Hubble sequence of red bulges and blue disks. The remainder of the sample are what we have termed "blue nucleated galaxies" - galaxies with concentrated blue components that nearly always have large, highly asymmetric, residuals from the fitting process. While the parameters of the fits give that these are "bulge-dominated" galaxies, the large and asymmetrical residuals have led us to interpret these as either irregular galaxies with off-centered star-formation or as systems that are the result of mergers or interactions. These galaxies are presumably the "peculiar" or "irregular" class of galaxies shown to have a steep number count in the HST Medium Deep Survey by Glazebrook et al. (1995) and Driver et al. (see these proceedings).

In the $50 \%$ of galaxies that are both well-represented by bulge-plus-disk models and have $B / T<0.5$, the disk surface brightnesses are significantly brighter (by about one magnitude) than seen in local samples, indicating a higher star-formation rate per unit area (see Fig 3 ).

Thus our present interpretation of the imaging data is that at least two processes are occurring to produce the changes in the blue galaxy luminosity function - the disk brightening in normal regular galaxies and the emergence of a populatiun of relatively bright "irregular" galaxies whose nature is less clear. As our sample of galaxies with both HST imaging and spectroscopically determined redshifts grows (we should have 350 galaxies by the end of HST Cycle 5) we should be able to study statistical distribution functions such as the disk size function, the B/T distribution, the 


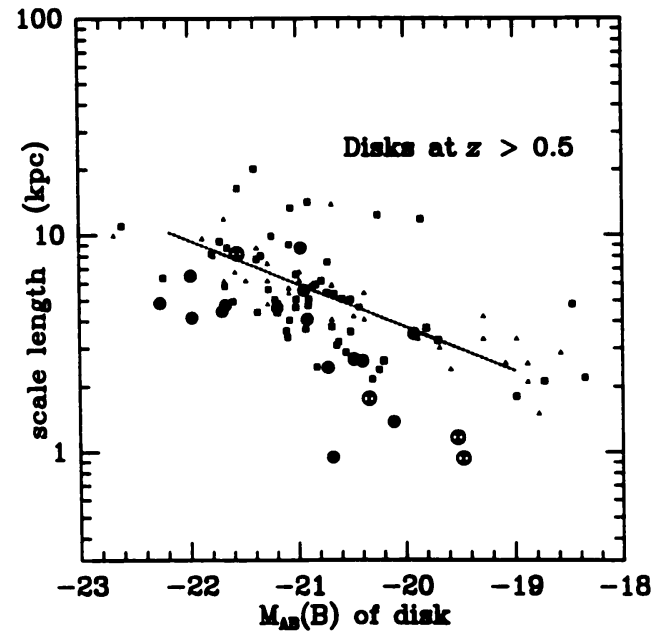

Figure 3. The disk scalelength as a function of disk luminosity for the $z>0.5$ galaxies that have $B / T<0.75$ (computed for $H_{0}=50 \mathrm{kms}^{-1} \mathrm{Mpc}^{-1}$ and $q_{0}=0.5$ ). Small symbols are data on local galaxies from Kent (1985) and van der kruit (1987). Dashed line is the Freeman "law" $\mu(B)=21.65 \mathrm{mag} \operatorname{arcsec}^{-2}$. The galaxy disks at $z>0.5$ are of systematically higher surface brightness. See Schade et al. (1995) for details.

bulge luminosity function, etc.. This will enable us to constrain much better the range of allowable scenarios for the evolution of field galaxies.

\section{References}

Broadhurst, T.J., Ellis, R.S., Shanks, T., 1988, MNRAS, 235, 827

Coleman, G. Wu, C., Weedman, D., 1980, ApJS, 43, 393

Colless, M.M., Ellis, R., Taylor, K., Hook, R.N., 1990, MNRAS, 244, 408

Cowie, L.L., Songaila, A., Hu, E.M., 1991, Nature, 354, 460

Crampton, D., Le Fevre, O., Lilly, S.J., Hammer, F., 1995, ApJ, 455, in press

Davis, M., Peebles, P.J.E., 1983, ApJ, 267, 465

Fisher, B.F., Davis, M., Strauss, M., Yahil., A., Huchra, J., 1994, MNRAS, 266, 50.

Fomalont, E.B., Windhorst, R., Kristian, J.A., Kellerman, K., 1991, AJ, 102, 1258

Hammer, F., Crampton. D., Le Fevre, O., Lilly, S.J., 1995a, ApJ, 455, in press

Hammer, F., Crampton, D., Lilly, S., Le Fevre, O., Kenet, T., 1995b, MNRAS, in press Hudon, J.D., Lilly, S.J., 1996, ApJ, in press.

Kent, S., 1985, ApJS, 59, 115

Koo, D.C., Kron, R.G., 1992, ARAA, 30, 613.

Koo, D.C., Gronwall, C., Bruzual, G., 1993, ApJ(Lett), 415, L21

Le Fevre, O., Crampton. D., Lilly, S.J., Hammer, F., Tresse, L., 1995a, ApJ, 455, in press, Le Fevre, O., Hudon, D., Lilly, S.J., Crampton. D., Hammer, F., 1996, ApJ, in press Lilly, S.J., 1993, 411, 501

Lilly, S.J., Le Fevre, O., Crampton. D., Hammer, F., Tresse, L., 1995a, ApJ, 455, in press Lilly, S.J., Hammer, F., Le Fevre, O., Crampton. D., 1995b, ApJ, 455, in press

Lilly, S.J., Tresse, L., Hammer, F., Le Fevre, O., Crampton, D., 1995c, ApJ, 455, in press Loveday, J., Peterson, B.A., Efstathiou, G., Maddox, S.J., 1992, ApJ, 390, 338.

Marzke, R.O., Geller, M.J., Huchra, J.P., Corwin, H., 1994, ApJ, 428, 43.

Schade, D.J., Lilly, S.J., Crampton, D.C., Hammer, F., Le Fevre, O., Tresse, L.; 1995, ApJLett, 451, 1 . 
Schade, D.J., Crampton, D.C., Le Fevre, O., Hammer, O., Lilly, S.J.; 1996, MNRAS, in press.

Tinsley, B.M., Gunn, J.E., 1976, ApJ, 203, 52.

Tresse, L., Hammer, F., Le Fevre, O., Proust, D., 1993, A\&A, 277, 53

van der Kruit, P., 1987, A\&A, 173, 59

\section{DISCUSSION:}

Simon White: Your conclusion that the early-type galaxies have the same rest-frame B-luminosity function at $z \sim 1$ as at $z \sim 0$ would, if correct, imply strong evolution of the population. This is because if all early-type galaxies were formed at $z>1$, we would expect a fading of at least a magnitude at rest-B between $z \sim 1$ and $z \sim 0$ due to the passive evolution of their stellar populations.

Simon Lilly: You raise an important point that I should have emphasized more in my talk - it is discussed at length in Lilly et al. (1995c). It is important to remember that the amount of passive evolution expected is very sensitively dependent on the poorly constrained slope of the i.m.f. in early-type stellar systems. Ideally, we will soon get some observational constraints on this from studying the fundamental plane (or projections thereof) at high redshift.

Ortwin Gerhard: From dynamical considerations, one might expect that some bulges formed by a bar instability followed by a bending instability scattering disk stars out of the plane. If this happened, then an observational signature would be a blue bulge. Do you see any objects in your sample that might plausibly be interpreted in this way.

Simon Lilly: Our sample observed with HST is still small (32 galaxies at $z>0.5$. Of these, $70 \%$ can be decomposed into a red bulge and a blue disk. The remainder have compact blue coponents which may conceivably be "bulges". However, these galaxies are almost always associated with large and asymmetric residuals in our two-dimensional fitting algorithm, and so we have tended to interpret them so far as either irregular galaxies with off-centered dominant HII regions or as the result of mergers or interactions (see Schade et al 1995). So this question is still open, I think.

Alvio Renzini: Qualitatively, it looks like the clusters and field do the same thing. Is this true quantitatively? i.e. is the evolution of the LF of blue galaxies in the field sufficient to "explain" the B-O effect?

Simon Lilly: That has not been looked at in detail. My impression is that, at a given redshift, the B-O effect may involve more luminous blue galaxies than the field evolution. However, as you say, there are indeed many qualitative similarities. One related result is the fact that the clustering properties of intrinsically red and blue galaxies are the same at high redshift, implying that the red-blue spatial segreation at low redshift has been eliminated. 